\title{
A case for psychosocial interventions to prevent mental illness
}

Tom K. J. Craig (1)

COMMENTARY ON... THE PSYCHOSIS RISK TIMELINE ${ }^{\dagger}$

\begin{abstract}
SUMMARY
The development of effective preventions for psychosis is hindered by conceptual challenges underlying diagnosis and the fact that few of the many biological risk factors identified to date are sufficiently well understood to form the basis of a targeted intervention. On the other hand, a great deal is known of the psychosocial conditions that increase the lifetime risk of most mental illnesses: surely enough to justify better resourcing of interventions focused on antenatal care and the emotional well-being of children from the early years through adolescence, where as much as a half of all mental ill health has its roots.
\end{abstract}

\section{DECLARATION OF INTEREST}

None.

\section{KEYWORDS}

Psychotic disorders; prevention; schizophrenia.

The three articles by Romain and colleagues in this issue (Romain 2019a, 2019b, 2019c) provide an excellent overview of the risk factors implicated in the cause of psychosis, which are thus potential targets for prevention. Preventive interventions can be either universal, in so far as some approaches benefit everyone, including those at risk, or targeted on a subpopulation because they are considered vulnerable to a specific disorder. Any targeted intervention must be clear about three things - the disorder to be prevented, the risk factor(s) to focus on and which approach to intervention is feasible at the time.

\section{The target disorder}

Psychosis is a portmanteau term encompassing several disorders that, in whole or in part (i.e. diagnostic entities or constituent symptoms), exist on a continuum shading off into non-pathological experiences. It is unclear whether the factors identified in these three articles might apply to a diagnosis, symptom, function or even some psychological construct or behavioural dimension, such as 'reward' or 'learning', that may underpin several disorders. The ultra-high-risk mental state may be a helpful foreshadowing of preventable disorder or something that is potentially a target of prevention itself.

\section{The target risk factors}

Looked at in the light of this complexity, it might seem surprising if any clear targets for prevention were to emerge. A genetic association may apply to just one of the several psychotic disorders encompassed by current diagnostic labels or to just one symptomatic or functional component of the condition. Knowledge of increased risk conferred by a particular gene or cluster of genes only takes us a little way to prevention without an understanding of the effects of the gene on cellular function. Ultimately, I suppose, some sort of genetic propensity score as a summation of small effects might have individual applicability but that is still a long way from knowing what to do about it.

Some biological risks look a bit closer to offering targets for preventive intervention, as for example the observation of an association between maternal inflammation during pregnancy and later risk of autism and psychosis in the offspring. It has been suggested that this is mediated by elevated levels of the pro-inflammatory cytokine interleukin-6 (Smith 2007). But even assuming that this mechanism is established for certain, there is still a way to go to develop a useful intervention (blocking interleukin- 6 would not be helpful as it is critically important for normal brain development, so that the benefit in terms of psychosis would be outweighed by wider developmental harms).

Nevertheless, even in the absence of a full understanding of the biology, there is much we can do to tackle the powerful effect of the environment. The incidences of schizophrenia, other psychoses, ultrahigh-risk status and even 'psychosis-like' experiences in the general population are elevated in
Tom K. J. Craig, MBBS, PhD, FRCPsych, is Emeritus Professor of Social Psychiatry at King's College London and an honorary consultant psychiatrist with South London and Maudsley NHS Foundation Trust, London, UK. A past president of the World Association of Social Psychiatry, his research interests include alternatives to the hospital asylum, services for first-episode psychosis and novel psychosocial treatments for psychosis.

Correspondence Professor Tom K. J. Craig, Health Service and Population Research Department, Institute of Psychiatry, Psychology and Neuroscience, De Crespigny Park, London SE5 8AF, UK. Email: thomas.craig@kcl.ac.uk

First received 10 Jun 2019 Accepted 14 Jun 2019

\section{Copyright and usage}

(C) The Royal College of Psychiatrists 2019

${ }^{\dagger}$ See this issue. 
people who have been exposed to traumatic situations, including childhood maltreatment, particularly involving sexual abuse, but also physical trauma and protracted bullying at school. Migrants and their offspring are at higher risk of psychosis, as are people living in densely packed, economically deprived urban areas. But these risk factors are far from specific to psychosis and devilishly difficult to do anything about. They are implicated across mental disorders, being a key component of the aetiological pathway for depression, anxiety, self-harm, substance misuse and personality disorder. To complicate things even further, not only are some risks exacerbated by the presence of others but, to be taken seriously, we need to understand how they play out across the many years that lie between their occurrence and the development of the target disorder (not entirely impossible, as much is now known about the links between childhood adversity, social function in adolescence, patterns of attachment and later depressive disorder, for example).

\section{The intervention}

While it may seem that targeting specific risk factors is the most attractive option, in fact universal strategies have a key part to play because many of the psychosocial risks are themselves manifestations of wider inequalities in income, education and housing conditions and relevant for multiple disorders (Marmot 2010; Burstow 2018). Sometimes a universal approach is also more acceptable because interventions targeted on specific individuals can arouse fears of stigmatisation and because those most likely to benefit from the intervention may also be the least willing to accept it.

Beyond the implementation of society-wide policies to address inequality, there is also the possibility of interventions that are somewhat more focused, even if not specific for psychosis. Prevention of obstetric complications, ensuring adequate maternal nutrition and reducing alcohol, tobacco and substance use are obvious targets, with some clear evidence that these risks can be reduced. Similarly, there are a number of programmes aimed at promoting good parenting and reducing the occurrence of childhood maltreatment, as well as school-based programmes that reduce levels of bullying, especially where the intervention has been delivered across several years and involved children, teachers and parents (for a summary of evidence see Burstow et al, 2018). Applying these to parents with mental disorders is an attractive option, as these interventions have been shown to reduce later incidence of mental disorder in their children by as much as 40\% (Siegenthaler 2012).

\section{Conclusions and implications for psychiatry}

Although we are still some way off devising a preventive intervention specifically for psychosis, there is surely enough evidence to justify better resourcing of universal prevention particularly focused on the prenatal period and throughout adolescence, when some of the greatest risks for future mental ill health have been identified. There are plausible suggestions of how this might be achieved and of the likely benefits such an approach might bring to society as a whole but, as ever, the challenge is one of achieving sustained investment in programmes that may not reveal their major effects for many years.

\section{References}

Burstow P, Newbigging K, Tew J, et al (2018) Investing in a Resilient Generation: Keys to a Mentally Prosperous Nation. University of Birmingham.

Marmot M (2010) Fair Society, Healthy Lives: The Marmot Review. Strategic Review of Health Inequalities in England Post 2010. Marmot Review.

Romain K, Eriksson A, Onyon R, et al (2019a) The psychosis risk timeline: can we improve our preventive strategies? Part 1: early life. BJPsych Advances, 25.

Romain K, Eriksson A, Onyon R, et al (2019b) The psychosis risk timeline: can we improve our preventive strategies? Part 2: adolescence and adulthood. BJPsych Advances, 25

Romain K, Eriksson A, Onyon R, et al (2019c) The psychosis risk timeline: can we improve our preventive strategies? Part 3: primary common pathways and preventive strategies. BJPsych Advances, 25.

Siegenthaler E, Munder T, Egger M (2012) Effect of preventive interventions in mentally ill parents on the mental health of the offspring: systematic review and meta-analysis. Journal of the American Academy of Child and Adolescent Psychiatry, 51: 8-17.

Smith S, Li J, Garbett K, et al (2007) Maternal immune activation alters fetal brain development through interleukin-6. The Journal of Neuroscience, 27: 10695-702. 